\title{
EN TORNO A LA CRONOLOGÍA DEL TEMPLO DE SAN DIONISIO DE JEREZ DE LA FRONTERA
}

Uno de los capítulos más interesantes en el desarrollo de la arquitectura cristiana medieval en Andalucía es el referido a los templos levantados en Jerez de la Frontera, en los que la convivencia de formas del primer gótico castellano con motivos ornamentales procedentes del arte almohade que les van a conferir un aspecto sumamente original. Por desgracía, la casi total carencia de datos seguros acerca de la edificación de los mismos, unida a la pervivencia de fórmulas arcaizantes a lo largo de un dilatado período de tiempo, ha impedido establecer una línea cronológica fiable que permita determinar con exactitud la evolución de la arquitectura en esta ciudad desde la conquista cristiana hasta que la decisiva implantación de la estética de la catedral de Sevilla señale un nuevo y fructífero rumbo en el devenir de las formas arquitectónicas.

En este artículo nos vamos a acercar a uno de los monumentos fundamentales del grupo: la iglesia dedicada a San Dionisio, santo patrón de la ciudad. Se trata de un edificio extraordinariamente singular por la original y exuberante ornamentación que presentan los pilares y arcos de sus tres naves, las portadas de las capillas.y los vanos -ciegos o no- del hastial y de la mitad inferior de la famosa torre de la Atalaya, adosada al ábside del Evangelio, aunque propiedad municipal desde su construcción. El ábside principal y la bóveda del crucero, aún de estirpe gótica, pertenecen a una cronología claramente posterior.

Dado que hasta los años sesenta el interior del templo permaneció enmascarado por una reforma dieciochesca, la peculiar decoración de arcos y pilares no pudo ser analizada en los fundamentales trabajos de Diego Angulo e Hipólito Sancho de Sopranis ${ }^{1}$. Tras la decisiva restauración felizmente promovida por el padre Luis Bellido (aún hoy cura párroco) y culminada por el arquitecto Rafael Manzano, diferentes historiadores se han acercado al edificio para exponer sus particulares puntos de vista. Sin embargo, la cronología del monumento sigue sin ser precisada ${ }^{2}$. Pues bien, presentando una montea hasta ahora inédita existente en el interior de la torre, vamos ahora a intentar arrojar alguna luz al respecto.

La torre de la Atalaya, también conocida hasta el pasado siglo como «torre del reloj de la ciudad», se compone de dos cuerpos prismáticos de planta rectangular. El más grande se distribuye en dos pisos, mientras que el más pequeño -que se adosa a su muro occidental- alberga una escalera de caracol, que gira que gira en sentido contrario al de las agujas del reloj. Las estancias del interior se cubren mediante bóvedas de crucería simple, con plementería de ladrillo, cuyos nervios parten de columnillas suspendidas con capiteles vegetales y de mocárabes. En la inferior los nervios presentan bocel de remate plano, al igual que los de la bóveda de acceso meridional al templo. En la estancia superior, ornada al exterior con cardinas y ar-

${ }^{1}$ D. Angulo Íñiguez: Arquitectura mudéjar sevillana de los siglos XIII, XIV y XV, Sevilla, 1932, reed. 1983. H. Sancho de Sopranis: «Introducción al estudio de la arquitectura en Jerez», en Guión, Jerez, 1934. La primera de estas obras sigue estando vigente en líneas generales, pero el análisis de los templos jerezanos es claramente incompleto. Mucho más extensa y atinada en el estudio de éstos resulta la segunda, aun también con reparos.

${ }^{2}$ Para Rafael Cómez el templo de San Dionisio es una obra alfonsí, relacionable con diversos templos cordobeses y sevillanos. Alfonso Jiménez sitúa iglesia y torre entre 1449 y 1457, señalando -muy acertadamente- su relación con la interesantísima parroquia de El Salvador de Vejer de la Frontera. Carlos García afirma que se levantó en la segunda mitad del XIV, momento en el que sitúa la consolidación del foco «mudéjar» de Jerez, datando el cuerpo inferior de la torre a comienzos del XV. R. Cómez Ramos: Arquitectura alfonsí, Sevilla, 1979, p. 113-119. A. Jiménez Martín: «Arquitectura gaditana de época alfonsí», en Cádiz en el siglo XIII, Cádiz, 1983, p. 150. C. García Peña: Arquitectura gótica religiosa en la provincia de Cádiz. Diócesis de Jerez, tesis doctoral líida en la Universidad Complutense de Madrid en 1990, p. $402-406$. 


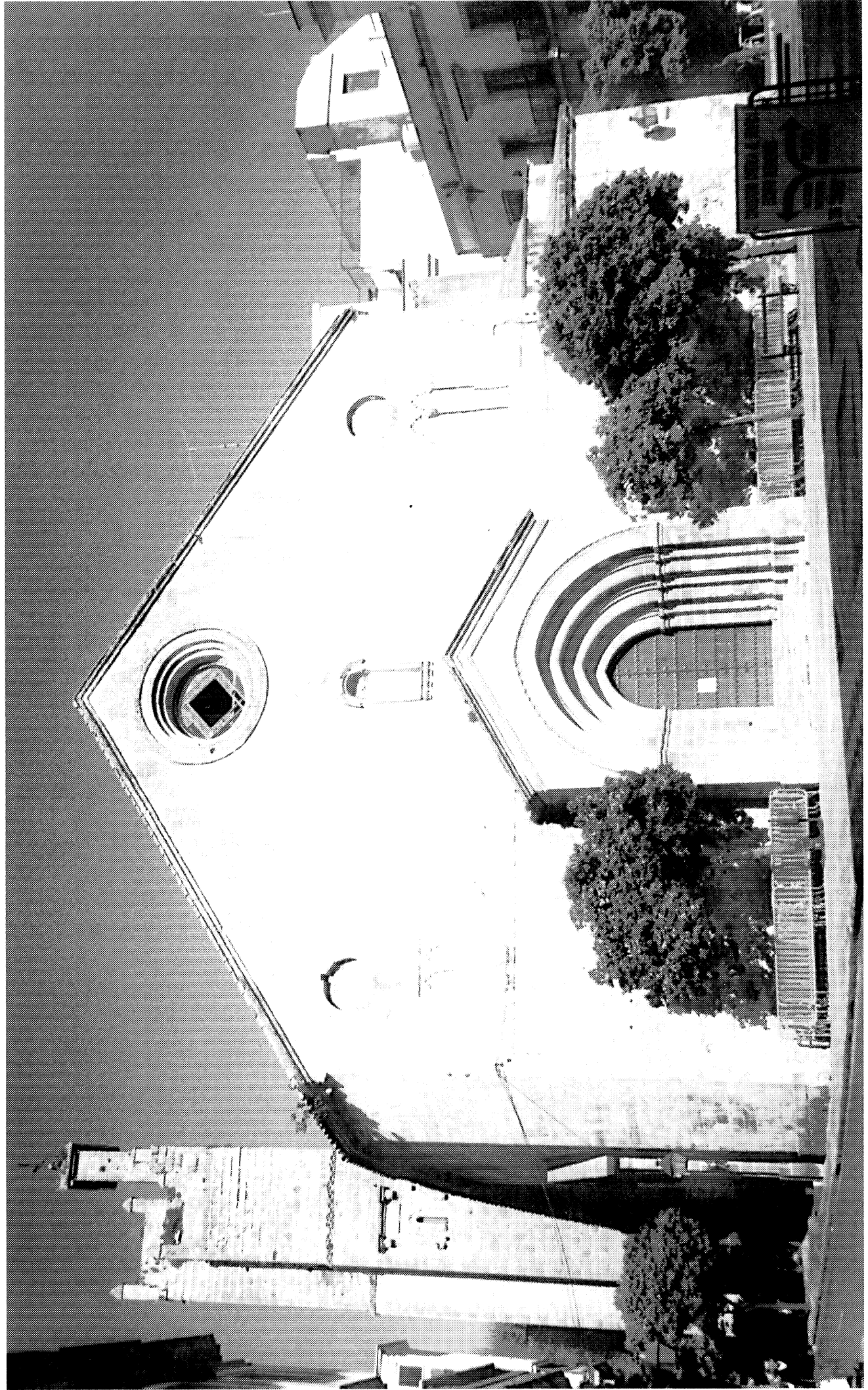

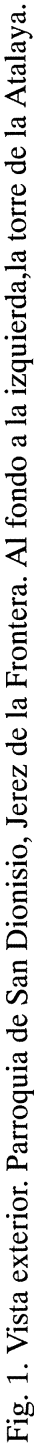


cos conopiales que delatan fechas muy avanzadas, se hallan flanqueados por dientes de sie$\mathrm{rra}^{3}$. La puerta que comunicaba la escalera con el exterior fue eliminada durante la restauración: el acceso se realiza hoy desde el interior de la iglesia ${ }^{4}$.

No hay duda de que el cuerpo superior se realizó bien entrado el siglo XV, como pone de manifiesto la ornamentación propia del gótico hispano tardío. Tampoco la hay de que el inferior es anterior a aquél. Frecuentemente se ha señalado el contraste en la ornamentación de los vanos y el cambio en la disposición interna de la escalera; añádase ahora a estas circunstancias la parcial alteración de los modelos de los numerosos signos de cantería que aparecen grabados en los muros, cuyo estudio se presentará en otra ocasión. Lo que seguimos ignorando es la causa de la paralización de las obras.

La montea se encuentra situada en la mitad inferior de la escalera, en un descansillo que accede desde la misma hasta un hueco que permite contemplar de cerca la alta bóveda del primer cuerpo (aproximadamente en la hilada cuarenta y tres). Cerca encontramos dos esbozos del mismo motivo, probablemente también monteas. Consiste en un lazo en forma de ocho tallado con cierto volumen, con el trazo inferior resuelto en suaves curvas y el superior apuntado.

Pues bien, dicho tipo de lazo es precisamente el que aparece en el extremo inferior de los peculiares baquetones entrelazados que recubren cada una de las cuatro caras de los pilares de la iglesia. Aunque encontramos abundantes lacerías en otros lugares del edificio, la montea corresponde única y exactamente a los «ochos» de estos gruesos baquetones. Ello lleva a pensar que el cuerpo inferior de la torre pertenece a la misma campaña constructiva en la que se levantaron los pilares y arcos del templo.

Aunque este aspecto lo corrobora la identidad del repertorio decorativo (entrelazo, angrelado, mocárabes), se ha venido sosteniendo que toda la torre es posterior a la construcción de las naves. Tal hipótesis fue formulada en 1932 por Angulo, quien sagazmente reparó en que los muros laterales de la misma se habían formado recreciendo unos contrafuertes del muro del Evangelio ${ }^{6}$. Sin embargo, la montea aquí presentada parece contradecir esta afirmación ${ }^{7}$.

La contradicción se resuelve si establecemos que el ábside junto al que se levanta la torre es una reutilización parcial de una fábrica anterior. El análisis detallado del ábside (perfil de los nervios, disposición de los sillares, ventanas ocultas por el retablo de San Cayetano) nos confirmará en un próximo trabajo esta hipótesis ${ }^{8}$. Nada inhabitual que fueran parcialmente aprovechadas estructuras de un edificio primitivo (último tercio del XIII o primero del XIV) a la hora de levantar el espléndido conjunto que hoy contemplamos.

\footnotetext{
${ }^{3} \mathrm{La}$ aparición de dientes de sierra en la bóveda de un recinto ornado con los elementos referidos demuestra a las claras que en Jerez se sigue usando este motivo en fechas muy avanzadas, como ocurre en el templo de Santo Domingo. F. López Vargas-Machuca: «Un ejemplo de reutilización y asimilación de arquitectura almohade: la iglesia del convento de Santo Domingo de Jerez de la Frontera», en Actas del XI congreso del CEHA. (Valencia. Septiembre 1996), Valencia, 1998, p. 27-30.

${ }^{4}$ Hasta no hace muchas décadas este acceso se encontraba tapiado. Angulo: ob. cit., p. 160-162.

${ }^{5}$ Como ha señalado Carlos García, existe un cierto parecido entre el vano ciego de la cara oriental y los respaldos de la sillería alta del coro de la catedral de Sevilla, obra firmada por Nufro Sánchez en 1478. García: ob. cit., p. 383.

${ }^{6}$ Angulo: ob. cit., p. 160-162. Este autor señaló la relación entre los vanos de la torre y las puertecillas del ábside del templo de San Juan de los Caballeros, que data hacia 1420-23, pero hoy día no podemos apoyarnos en tal similitud para establecer precisiones cronológicas, ya que las mencionadas puertecillas son falsas. Sancho de Sopranis: ob. cit., p. 4

${ }^{7}$ También la contradice la ya referida similitud en la ornamentación: «Es verdad que la fábrica de la torre aparece claramente añadida a la principal de la iglesia contigua, pero son tantos los detalles que enlazan la torre con la iglesia misma, que hay que suponerlas muy poco separadas en el tiempo». C. Pemán: «Sobre la torre de San Dionisio de Jerez», en Academia, 1953-54, t. II, pp. 88-90. Recordemos que Angulo conoció el templo aún sin restaurar.

${ }^{8}$ López: "En torno a la arquitectura gótica andaluza en el siglo XVIII: el caso de Jerez de la Frontera", Actas del Congreso Sevilla, 1248 (Sevilla, noviembre 1998). En prensa.
} 



$m$

N

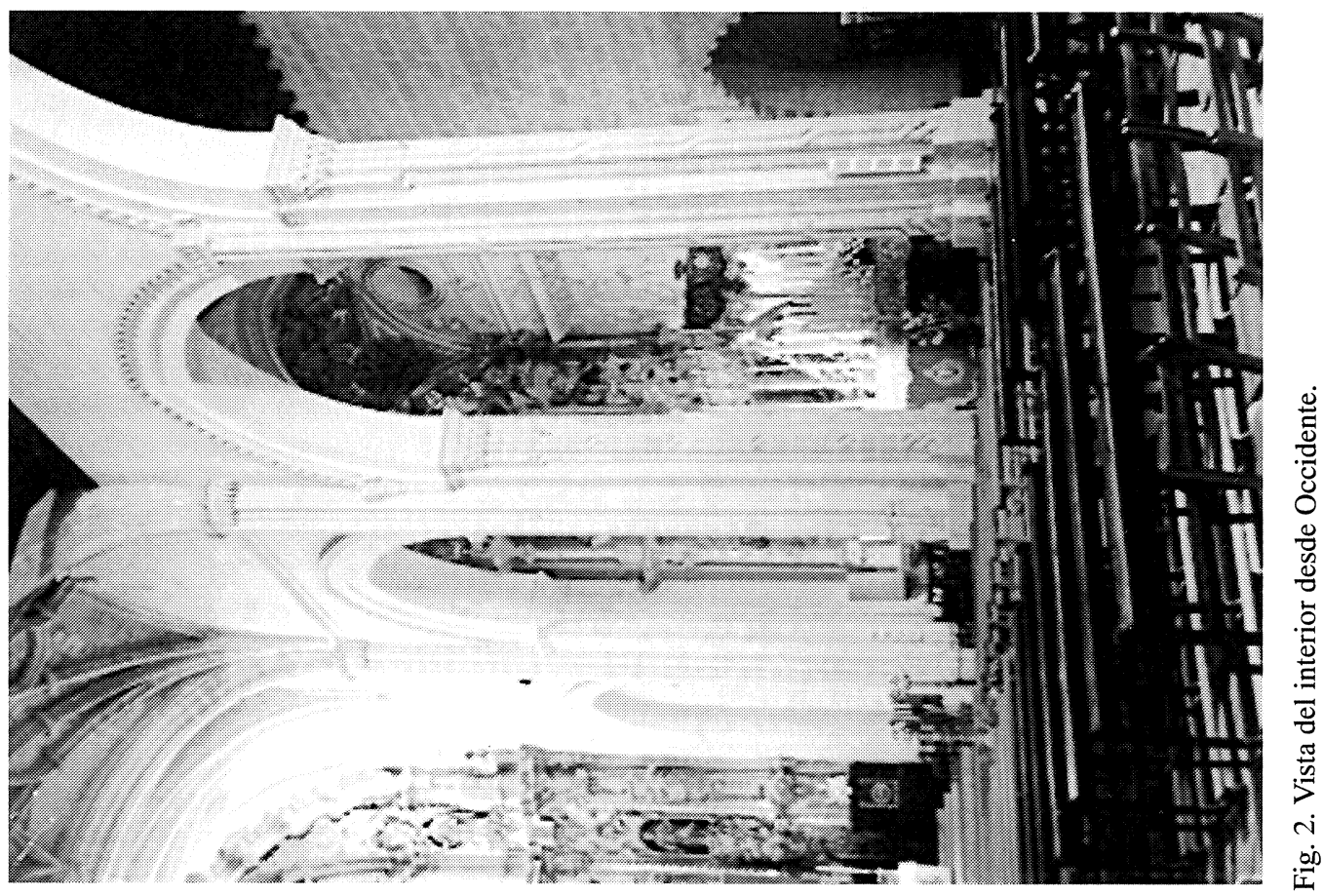


No conocemos ninguna fecha correspondiente a las obras medievales de nuestro edificio, salvo en lo que se refiere a la torre. Así, en las Actas Capitulares del Cabildo del 17 de marzo de 1447, se lee que «fue ordenado faser relox...» (por el Adelantado Per Afán de Ribera) «...e está çerca de fecha la torre para el dicho relox,...». Por tanto, tenemos la certeza de que en dicho momento ésta se encontraba en obras, lo que encaja con la afirmación de Bartolomé Gutiérrez - que no cita su fuente- según la cual fue levantada en 1449 , fecha que podría corresponder a la momentánea culminación de la empresa ${ }^{10}$. De esta manera tenemos un término ante quem para la construcción no sólo de la torre, sino también de los pilares y arcos de las naves ${ }^{11}$.

Luis de Grandallana señaló otra fecha: $1420^{12}$. Dado que no hemos localizado su fuente, no debemos caer en la tentación de establecer que ésta corresponde al primer cuerpo (y por ello a las naves), y que en 1447-49 lo que se levanta es el segundo, donde estuvo colocado el reloj. Además, resulta un momento temprano para la aparición en Jerez de formas propias del gótico tardío, de momento lo mejor es descartar esta hipótesis ${ }^{13}$.

Sea como fuere, podemos confirmar que las naves del templo y el primer cuerpo de su torre fueron construídos dentro de una misma empresa, no posterior a 1449, y probablemente dentro de la primera mitad del siglo $\mathrm{XV}$, reaprovechando elementos de una fábrica más antigua. De esta manera obtenemos un elemento de referencia a la hora de estudiar otros edificios de su órbita.

\section{FERNANDO LóPEZ VARGAS-MACHUCA} Licenciado en Historia del Arte

\section{OTRA PINTURA FIRMADA DE SALVADOR JORDÁN}

Un estudio de Alfonso E. Pérez Sánchez publicado en estas mismas páginas, atribuye verosímilmente a Salvador Jordán, pintor y grabador documentado en Madrid en 1708 y 1722, dos cuadros de tema religioso firmados «Jordán» ${ }^{1}$. Uno de ellos representa la Visión de la Virgen y el Niño por San Antonio de Padua y está además fechado en 1700; se conserva en el Santuario de Loyola y fue dado a conocer como obra de Luca Giordano². El otro lienzo representa la Inmaculada Concepción rodeada de ángeles y es propiedad del Ayuntamiento de Vitoria-Gasteiz. Se expuso en la muestra, «Barroco importado en Álava, escultura y pintura», que tuvo lugar en

${ }^{9}$ Archivo Municipal de Jerez de la Frontera, Actas Capitulares de 1447, fol. 24 v. Este documento fue publicado por A. Muñoz y Gómez: Historiógrafos y antigüedades de Xerez de la Frontera, Jerez, 1889, pp. 161-162. Posteriormente fue recogido por M. Esteve Guerrero: Jerez de la Frontera (Guía oficial de arte), Jerez, 1952, p. 122. Posiblemente en el mismo se base la certificación del Ayuntamiento de 1977 que Carlos García ha presentado según la cual la torre de la Atalaya figura en todos los inventarios municipales desde 1447. García: ob. cit., p. 406

${ }^{10} \mathrm{~B}$. Gutiérrez: Historia del estado presente y antiguo de la muy noble y muy leal Xerez de la Frontera, Jerez, 1886, (ed. facsímil: 1989), vol. 1, p. 312.

${ }^{11}$ No nos interesa aquí la fecha de 1457 que recogen diversos investigadores. Ésta fue aportada por Mesa Xinete, quien presentó el testamento de un tal Juan Román, dictado entonces, en el cual expresaba haber comprado una sepultura «junto a el altar mayor, que por entonces se estaba labrando» (el subrayado es nuestro). Como vemos, esta expresión no tiene que ver con la erección de las naves. F. Mesa Xinete: Historia sagrada y política de Xerez de la Frontera, Jerez, 1888, pp. $181-82$.

${ }^{12}$ L. de Grandallana y Zapata: Noticia histórico-artística de algunos de los principales monumentos de Jerez, Jerez, 1885 (ed. facsímil: 1989), p. 43 .

${ }^{13}$ En la portada occidental de la parroquia de la $\mathrm{O}$ de Sanlúcar de Barrameda, directamente vinculada al círculo jerezano, se entremezclan elementos decorativos que encontramos en los dos cuerpos de la torre de la Atalaya. Desgraciadamente no conocemos aún la cronología exacta de la obra sanluqueña. Ofrece una hipótesis interesante H. Sancho de Sopranis: «Un monumento mudéjar poco conocido de la Baja Andalucía: Santa María de la O de Sanlúcar de Barrameda», en Mauritania, marzo de 1943, n. 184, pp. 75-79.

' Alfonso E. Pérez Sánchez, «Salvador Jordán, pintor madrileño», Archivo Español de Arte, N. 271 (1995), pp. 299-303.

${ }^{2}$ Ignacio Cendoya Echániz y Pedro María Montero Esteban, «Nueva aportación a la obra de Lucas Jordán», Boletín del Seminario de Estudios de Arte y Arqueología de la Universidad de Valladolid, Vol. LVIII (1992), pp. 379-381. 